\title{
Effect of Nitrogen Implantation on the Performance of TOHOS Total Ionizing Dose Radiation Sensor Device
}

\author{
Wen-Ching Hsieh, ${ }^{*}$ Hao-Tien Daniel Lee, ${ }^{1}$ Fuh-Cheng Jong, ${ }^{2}$ and Shich-Chuan $\mathrm{Wu}^{3}$ \\ Department of Opto-Electronic System Engineering, Minghsin University of Science and Technology, \\ Xinxing Road 1, Xinfeng 30401, Taiwan \\ ${ }^{1}$ Treasure Giant Technology Inc. 3F-1, 42, Lyushuei Road, Hsinchu 30068, Taiwan \\ ${ }^{2}$ Electronic Engineering Department, Southern Taiwan University of Science and Technology, \\ 1, Nan-Tai Street, Yungkang District, Tainan 710, Taiwan \\ ${ }^{3}$ National Nano Device Laboratories, No. 26, Prosperity Road 1, Hsinchu Science Park, Hsinchu 30078, Taiwan
}

(Received September 1, 2015; accepted January 19, 2016)

Keywords: high k, sensor, TID, SONOS, SOHOS, MOS, radiation

The nitrogen-implanted titanium nitride-silicon oxide-hafnium oxide-silicon oxide-silicon (hereafter, N-TOHOS) device can be a candidate for total ionizing dose (TID) radiation sensor application. In this study, we mainly focus on $\mathrm{N}$ implantation for the improvement of the TID radiation-induced charging effect performance of the N-TOHOS device. The TID radiation-induced charging effect performance of the N-TOHOS device is improved by the implantation of nitrogen in the $\mathrm{HfO}_{2}$ trapping layer. Experimental data show that the radiation-induced charge density of a typical N-TOHOS device is approximately 4-5 times that of a conventional metal-oxide-nitrideoxide-silicon (MONOS) device reported previously. The charge retention reliability after $5 \mathrm{mrad}$ gamma irradiation of the N-TOHOS device with a high implantation dose and a low implantation energy can be improved. The N-TOHOS device described in this study has demonstrated its potential for nonvolatile TID radiation sensing application in the future.

\section{Introduction}

The total ionizing dose (TID) radiation effect is a major application concern for the operation of electronic devices in advanced X-ray lithography semiconductor manufacturing processes and outer space applications, as well as in other harsh environments such as accelerators, where highand low-energy particles exist. After gamma irradiation, it is well known that the metal-silicon dioxide-silicon (MOS) structure shows a build-up of positive charges at the silicon-silicon dioxide $\left(\mathrm{Si}-\mathrm{SiO}_{2}\right)$ interface and an interface state occurs in the structure. ${ }^{(1)}$ The radiation-induced charging effects of a metal-nitride-silicon dioxide-silicon (MNOS) device with stacked insulation layers composed of silicon nitride and silicon dioxide have been reported. ${ }^{(2)}$ TID radiation-induced charging effects in traditional silicon-silicon dioxide-nitride-silicon dioxide-silicon (SONOS) nonvolatile memory (NVM) devices have been studied. ${ }^{(3-5)}$ Until now, little is known about the radiation response of SONOS-like devices with a high-k gate dielectric structure. ${ }^{(6,7)}$ High-k gate dielectrics have been used for reducing the transistor gate leakage current in advanced nanoscale complementary metal-oxide-semiconductor (CMOS) devices. ${ }^{(8-10)}$ Recently, the conventional

"Corresponding author: e-mail: wchsieh@must.edu.tw 
SONOS flash memory has been replaced with the SOHOS memory device. However, the SOHOS device has very poor data retention characteristic. ${ }^{(8-10)}$ The radiation-induced charging effect of a few hafnium-based MOS devices have been reported. ${ }^{(6,7)}$ However, the data retention reliability of the hafnium-based SONOS-like device after gamma irradiation has not been well studied and it will be the main focus of this study.

To improve the charge retention reliability of the hafnium-based SONOS-like device after gamma irradiation, a nitrogen-implanted titanium nitride-silicon oxide-hafnium oxide-silicon oxide-silicon (hereafter, N-TOHOS) device is fabricated. The N-TOHOS device as illustrated in Fig. 1 is characterized by its delta $V_{\mathrm{T}}$ performance as a potential candidate TID radiation sensor in this study. The electrical performances of N-TOHOS devices with various implantation doses and energies after gamma irradiation, including charge storage density, gate leakage current, $V_{\mathrm{T}}$ stability, and retention time, are the main subjects of this study.

\section{Experimental Details}

N-TOHOS devices obtained under various nitrogen implantation conditions are listed in Table 1. The various devices denoted as N1-TONOS to N7-TOHOS were used throughout this study. N1TONOS with a nitride-trapping layer and N2-TOHOS without nitrogen implantation were used for



Fig. 1. (Color online) Cross-sectional view of N-TOHOS device.

Table 1

$\mathrm{N}-\mathrm{TOHOS}$ devices prepared under various implantation conditions.

\begin{tabular}{|c|c|c|c|c|c|c|c|}
\hline & N1-TONOS@ & N2-TOHOS@ & N3-TOHOS & N4-TOHOS & N5-TOHOS & N6-TOHOS & N7-TOHOS \\
\hline Trapping layer & $\mathrm{Si}_{3} \mathrm{~N}_{4}$ & $\mathrm{HfO}_{2}$ & $\mathrm{HfO}_{2}$ & $\mathrm{HfO}_{2}$ & $\mathrm{HfO}_{2}$ & $\mathrm{HfO}_{2}$ & $\mathrm{HfO}_{2}$ \\
\hline $\begin{array}{l}\mathrm{N} \text { implantation } \\
\text { dose }\left(\mathrm{cm}^{-2}\right)\end{array}$ & No & No & $\begin{array}{l}1 \mathrm{E}+13 \\
(\mathrm{LD})^{*}\end{array}$ & $\begin{array}{l}1 \mathrm{E}+14 \\
(\mathrm{MD})^{*}\end{array}$ & $\begin{array}{l}1 \mathrm{E}+15 \\
(\mathrm{HD})^{*}\end{array}$ & $\begin{array}{l}1 \mathrm{E}+15 \\
(\mathrm{HD})^{*}\end{array}$ & $\begin{array}{l}1 \mathrm{E}+15 \\
(\mathrm{HD})^{*}\end{array}$ \\
\hline $\begin{array}{c}\mathrm{N} \text { implantation } \\
\text { energy }(\mathrm{keV})\end{array}$ & No & No & $5(\mathrm{LE})^{\#}$ & $5(\mathrm{LE})^{\#}$ & $5(\mathrm{LE})^{\#}$ & $7(\mathrm{ME})^{\#}$ & $9(\mathrm{HE})^{\#}$ \\
\hline
\end{tabular}

@: N1-TONOS with nitride-trapping layer and N2-TOHOS without N implantation are used for comparison.

*LD, low dose; MD, medium dose; HD, high dose.

${ }^{*}$ LE, low energy; ME, medium energy; HE, high energy. 
comparison. The N-channel TOHOS devices were fabricated at the Taiwan National Nano Device Laboratories (NDL, Hsinchu, Taiwan). Starting wafers were 6" silicon having a (100) orientation with boron-doped substrates and a resistivity of $15-25 \Omega \cdot \mathrm{cm}$. The tunneling silicon oxide $\left(\mathrm{SiO}_{2}\right)$ was formed by using an advanced clustered vertical furnace on the wafers and its thickness was 3-5 nm, which was measured using a spectroscopic ellipsometer. After the tunneling oxide formation, $\mathrm{Si}_{3} \mathrm{~N}_{4}(100-200 \AA)$ was deposited as charge-trapping layers by low-pressure chemical vapor deposition (LPCVD) for the N1-TONOS device. For the TOHOS devices, $\mathrm{HfO}_{2}$ films were deposited as the charge-trapping layers using a metal organic chemical vapor deposition (MOCVD) system at $400-550^{\circ} \mathrm{C}$ for an approximate thickness of $10-20 \mathrm{~nm}$. The precursor Hf (tert-butoxy) $(\mathrm{mmp})_{2}$ was used in the MOCVD system. The blocking oxide (100-200 $\AA$ thickness) was deposited by LPCVD using tetra ethyl oxysilane (TEOS) $\left[\mathrm{Si}\left(\mathrm{OC}_{2} \mathrm{H}_{5}\right)_{4}\right]$. After blocking oxide deposition, the nitrogen implantation into $\mathrm{HfO}_{2}$ was carried out at low energy (LE, $5 \mathrm{keV}$ ), medium energy (ME, $7 \mathrm{keV}$ ), and high energy (HE, $9 \mathrm{keV})$, with low dose (LD, $1 \mathrm{E} 13$ atoms $\left./ \mathrm{cm}^{2}\right)$, medium dose (MD, $1 \mathrm{E} 14$ atoms $\left./ \mathrm{cm}^{2}\right)$, and high dose (HD, 1E15 atoms $\left./ \mathrm{cm}^{2}\right)$. The control gate with a $200-400 \mathrm{~nm}$ TiN metal gate was formed by DC sputtering for all samples. The resulting structure is illustrated in Fig. 1. After gate patterning, source and drain were formed by implantation with arsenic atoms, which were activated at $900{ }^{\circ} \mathrm{C}$ for $30 \mathrm{~s}$. For comparison, all the devices listed in Table 1 have the same thicknesses of tunneling oxide, trapping oxide, and blocking oxide layer.

In this work, the $V_{\mathrm{T}}$ shifts due to gamma irradiation on the N-TOHOS devices prepared by various implantation methods were measured. The gamma irradiation was performed on the N-TOHOS devices with negative gate bias stress (NVS) $\left(V_{\mathrm{G}}=-1 \mathrm{~V}\right)$ using a ${ }^{60} \mathrm{Co}$ irradiator source at room temperature. The $V_{\mathrm{T}}$ changes of the N-TOHOS devices prepared by various implantation methods were examined after the gamma irradiation. $V_{\mathrm{T}}$ was measured at room temperature using a HP4156A parameter analyzer. The experimental results of gate leakage current under various gate voltages $\left(I_{\mathrm{G}}-V_{\mathrm{G}}\right)$ were obtained using a computer-controlled HP4156 parameter analyzer. The experimental results of gate capacitance applied with various gate voltages $\left(C_{\mathrm{G}}-V_{\mathrm{G}}\right)$ were obtained using a computer-controlled HP4284 parameter analyzer, and the $C_{\mathrm{G}}-V_{\mathrm{G}}$ curves were obtained by sweeping $V_{\mathrm{G}}$ under zero source-and-drain bias condition at room temperature.

Figure 2 shows the charge generation and trapping states of the gate dielectric for the N-TOHOS device under NVS after gamma irradiation.

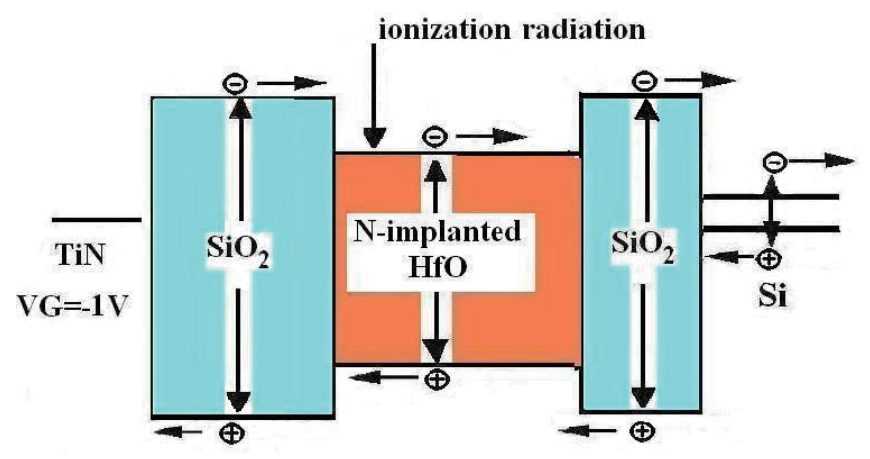

Fig. 2. (Color online) Charge generation and trapping states in N-TOHOS device under NVS after gamma irradation. 


\section{Results and Discussion}

\subsection{Radiation-induced charging effect of TOHOS after gamma irradiation}

Figure 3 shows the $I_{\mathrm{D}}-V_{\mathrm{G}}$ curve for a typical N5-TOHOS device with negative bias voltage ( $V_{\mathrm{G}}$ $=-1 \mathrm{~V}$ ) and processed with gamma irradiation up to $5 \mathrm{mrad}$ TID. A significant negative $V_{\mathrm{T}}$ shift of the N5-TOHOS device is observed in the figure, which is a result of the increase in positive charges in the trapping layer caused by ionization radiation. The negative $V_{\mathrm{T}}$ shift result agrees with those of previous studies. ${ }^{(5,6)}$ The radiation-induced negative $V_{\mathrm{T}}$ shift in the irradiated N5TOHOS device is induced by a combination of storage charge loss in the $\mathrm{HfO}_{2}$ trapping layer and a build-up of positive charges from the asymmetric trapping of electrons and holes in the trapping layer. ${ }^{(5,6)}$ As the N5-TOHOS device was irradiated by ionization radiation, the electron-hole pairs are generated throughout the gate insulation layer, and fractions of free electrons and holes escaped from the initial recombination process. The TID radiation-induced charging effect on the device is determined by the survival yield of electron-hole pairs, which is a portion of free holes and electrons that survived after the recombination process immediately following the excitation processes due to ionization radiation.

The $\mid$ delta $V_{\mathrm{T}} \mid$ of the N5-TOHOS device increases as a function of gamma TID, as indicated in Fig. 4. It also shows a quasi-linear correlation of |delta $V_{\mathrm{T}} \mid$ vs gamma radiation TID below $100 \mathrm{krad}$ in $\log$ scale, but $\mid$ delta $V_{\mathrm{T}} \mid$ increases more sharply after gamma irradiation at levels up to $100 \mathrm{krad}$ TID. ${ }^{(5,6)}$ This result is in agreement with those of previous studies. ${ }^{(5,6)}$

The $\mid$ delta $V_{\mathrm{T}} \mid$ and charge density comparisons after $5 \mathrm{mrad}$ TID gamma irradiation for various N-TOHOS devices shown in Table 1 are illustrated in Figs. 5(a) and 5(b), respectively. The trapped charge density can be calculated by the Terman method. ${ }^{(7)}$ The radiation-induced $V_{\mathrm{T}}$ shift of TOHOS without nitrogen implantation is more significant than that of TONOS, which results from more radiation-induced charges into the $\mathrm{HfO}_{2}$ trapping layer than into the $\mathrm{Si}_{3} \mathrm{~N}_{4}$ charging layer. Furthermore, the radiation-induced $V_{\mathrm{T}}$ shift increases with the implantation dose for the N-TOHOS device with the low implantation energy, as shown in Fig. 5(a). Note that the radiation-induced



Fig. 3. $I_{\mathrm{D}}-V_{\mathrm{G}}$ curve for N5-TOHOS device after 5 mrad TID gamma irradiation.

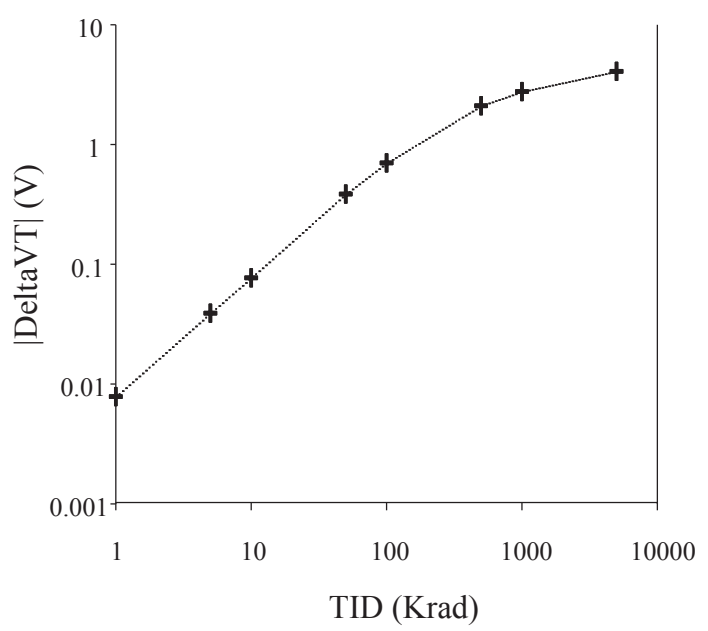

Fig. 4. $\mid$ Delta $V_{\mathrm{T}} \mid$ increase as a function of gamma radiation TID for N5-TOHOS device. 


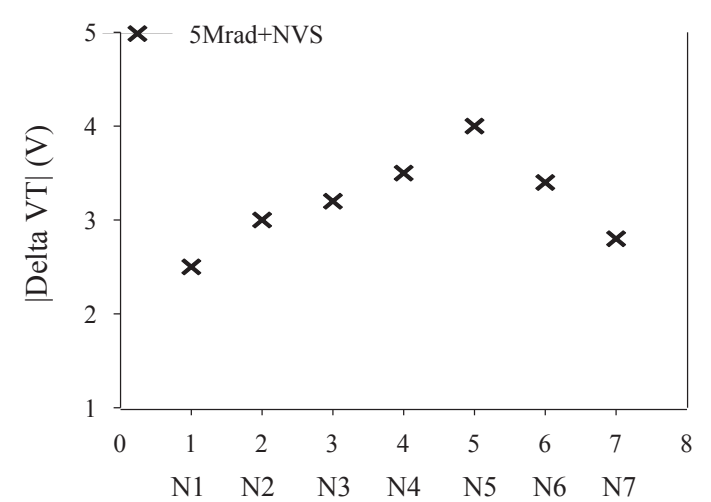

(a)



(b)

Fig. 5. (a) $\mid$ Delta $V_{\mathrm{T}} \mid$ comparison for N-TOHOS devices prepared by different implantation methods listed in Table 1 after 5 mad TID gamma irradiation. (b) Relative charge density comparison for various N-TOHOS devices after 5 mrad gamma irradiation.

charge density of the N5-TOHOS device with high-dose and low-energy implantation is 4.8 times larger than that of the TONOS device. Furthermore, The radiation-induced charging effect of the N5-TOHOS device is more significant than that of the metal-aluminum oxide-hafnium aluminum oxide-silicon oxide-silicon (MAHAOS) device reported previously.(6) The TID radiation-induced charging effect of the N-TOHOS device is improved by an increase in the amount of positive charges caused by gamma iradiation in the trapping layer, which is a result of the nitrogen atoms implanted in the $\mathrm{HfO}_{2}$ trapping dielectric. However, the radiation-induced $V_{\mathrm{T}}$ shift decreases with the implantation energy for the N-TOHOS device with high implantation dose, which is a result of the high-energy-implantation damage of the $\mathrm{HfO}_{2}$ trapping dielectric as shown in Fig. 5(a).

\subsection{Gate leakage current measurement}

Figure 6(a) and 6(b) show the gate leakage current for N-TOHOS devices prepared by various implantation methods after $10 \mathrm{krad}$ and $5 \mathrm{mrad}$ TID gamma irradiation, respectively. As shown in Fig. 6(a), the gate leakage current of TOHOS is extremely worse than that of TONOS after $10 \mathrm{krad}$ and 5 mrad TID gamma irradiation, which is because more interface traps of $\mathrm{HfO}_{2}$ lead to a larger gate current leakage after $10 \mathrm{krad}$ and $5 \mathrm{mrad}$ TID gamma irradiation. (7) The gamma irradiation could induce new crystallized phases and chemical structure change of $\mathrm{HfO}_{2}$, which would lead to the generation of trapped charges and leakage current in the $\mathrm{HfO}_{2}$ trapping layer.(7) However, by high-dose implantation of nitrogen into $\mathrm{HfO}_{2}$, the gate leakage current performance is markedly improved after $10 \mathrm{krad}$ and $5 \mathrm{mrad}$ TID gamma irradiation. After high-dose implantation, nitrogen atoms might be trapped in the interface traps of $\mathrm{HfO}_{2}$, leading to the improvement of the gate current leakage. This result suggests that high-dose nitrogen implantation is very effective for improving the gate leakage current of the N-TOHOS device after $10 \mathrm{krad}$ and $5 \mathrm{mrad}$ TID gamma irradiation. However, the gate leakage current performance of the N-TOHOS device by highenergy implantation is worse than that of TONOS after $5 \mathrm{mrad}$ TID gamma irradiation, which is a result of the high-energy-implantation damage of the blocking oxide and the charge leakage path after $5 \mathrm{mrad}$ gamma irradiation. 


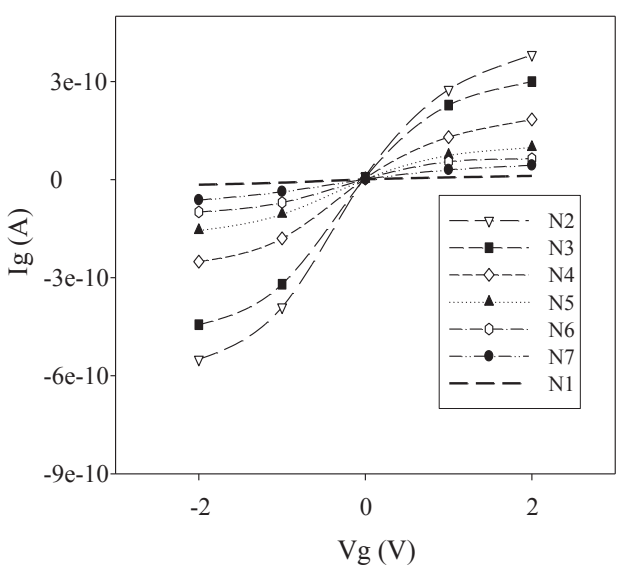

(a)

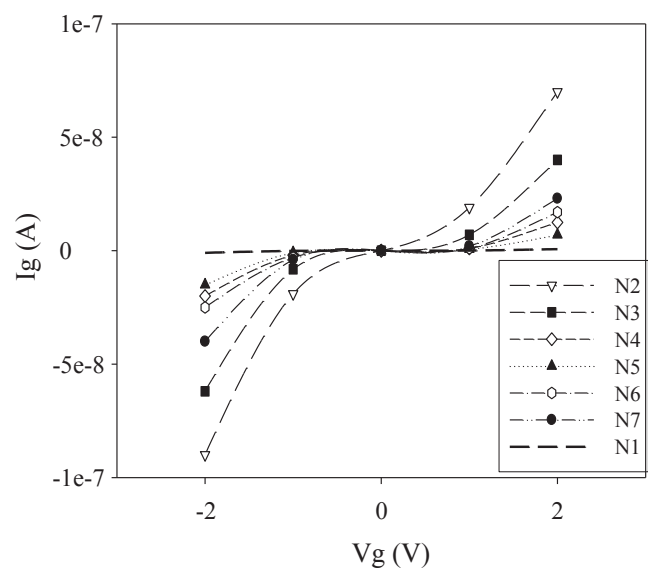

(b)

Fig. 6. (a) $\left|I_{\mathrm{G}}\right|\left(V_{\mathrm{G}}=-1 \mathrm{~V}\right)$ comparison for various N-TOHOS devices after 10 krad gamma irradiation. (b) $\left|I_{\mathrm{G}}\right|\left(V_{\mathrm{G}}\right.$ $=-1 \mathrm{~V}$ ) comparison for various N-TOHOS devices after $5 \mathrm{mrad}$ gamma irradiation.

\section{3 $\quad V_{\mathrm{T}}$ stability vs retention time}

In this section, the $V_{\mathrm{T}}$ stability and retention time of N5-TOHOS devices are discussed and these are the important electrical properties that need to be verified for their potential application in TID radiation sensors. Figure 7 shows the $V_{\mathrm{T}}$ stability vs retention time under NVS $\left(V_{\mathrm{G}}=-1\right.$ V) for the N5-TOHOS device after gamma irradiation. The upper curve is for $V_{\mathrm{T}}$ decay of N5TOHOS after $10 \mathrm{krad}$ TID gamma irradiation and the lower curve is for $V_{\mathrm{T}}$ decay of N5-TOHOS after $5 \mathrm{mrad}$ TID gamma irradiation. Note that the $V_{\mathrm{T}}$ decay over time of the N5-TOHOS device after gamma irradiation is a result of charge tunneling from the nitrogen-implanted $\mathrm{HfO}_{2}$ trapping layer. However, there is no noticeable decay trend of the $V_{\mathrm{T}}$ for the N5-TOHOS device after 5 mrad gamma irradiation. This trend can be attributed to a more effective charge recording ability inside the stack gate of the N5-TOHOS device after high-dose gamma irradiation.

Figures 8 and 9 show the $V_{\mathrm{T}}$ stability vs time under NVS $\left(V_{\mathrm{G}}=-1 \mathrm{~V}\right)$ for N-TOHOS devices prepared by various implantation methods after $10 \mathrm{krad}$ and $1 \mathrm{mrad}$ gamma irradiations, respectively. It is seen that the device with $\mathrm{HfO}_{2}$ as the charge-storage layer shows the worse charge retention reliability characteristic than $\mathrm{Si}_{3} \mathrm{~N}_{4}$. These results are consistent with the gate leakage results shown in Fig. 6(a) and mentioned earlier. Therefore, it seems that the device with $\mathrm{HfO}_{2}$ is degraded in terms of charge storage capacity by providing stored charges with a leakage path. The worse charge storage capacity in the TOHOS device may be attributed to tunneling leakage current induced by interface trap states. ${ }^{(8)}$ However, by applying high-dose and high-energy implantation of nitrogen into $\mathrm{HfO}_{2}$, the charge retention performance is markedly improved after $10 \mathrm{krad}$ and 1 mrad gamma irradiations. The charge retention reliability performance of the N-TOHOS device is improved owing to the deep traps induced by nitrogen implantation in the $\mathrm{HfO}_{2}$ trapping layer after $10 \mathrm{krad}$ and $1 \mathrm{mrad}$ gamma irradiations. . $^{(8,9)}$

Figure 10 shows the $V_{\mathrm{T}}$ stability versus time under NVS $\left(V_{\mathrm{G}}=-1 \mathrm{~V}\right)$ of N-TOHOS devices prepared by various implantation methods after $5 \mathrm{mrad}$ gamma irradiation. However, for high- 


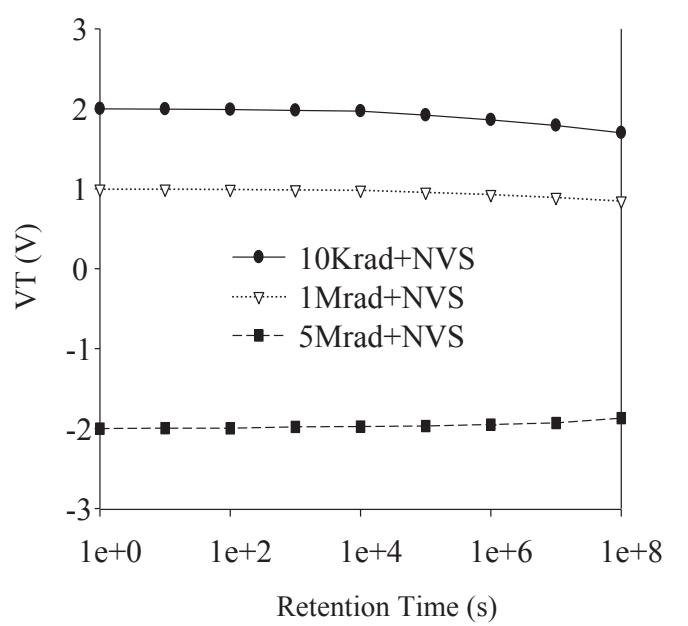

Fig. 7. $V_{\mathrm{T}}$ stability under $V_{\mathrm{G}}=-1 \mathrm{~V}$ for N5-TOHOS devices after gamma irradiation.

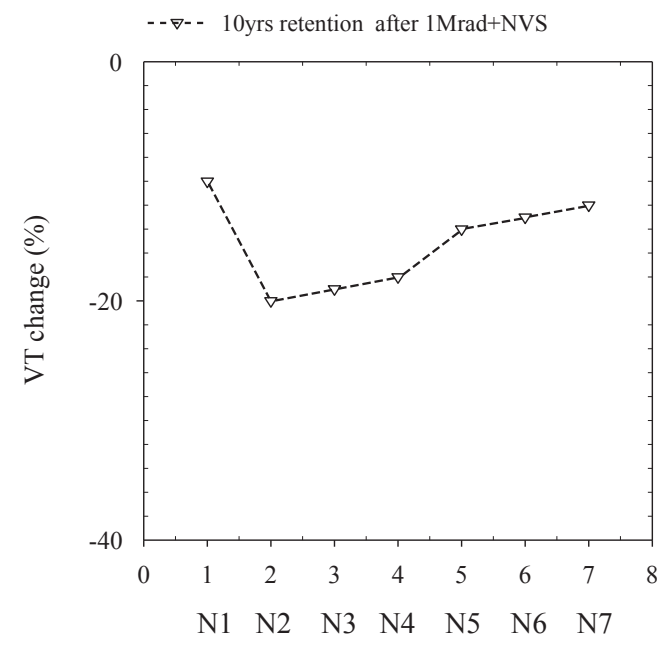

Fig. 9. $\quad V_{\mathrm{T}}$ change with 10-y retention time under $V_{\mathrm{G}}$ $=-1 \mathrm{~V}$ for various $\mathrm{N}-\mathrm{TOHOS}$ devices after $1 \mathrm{mrad}$ gamma irradiation.

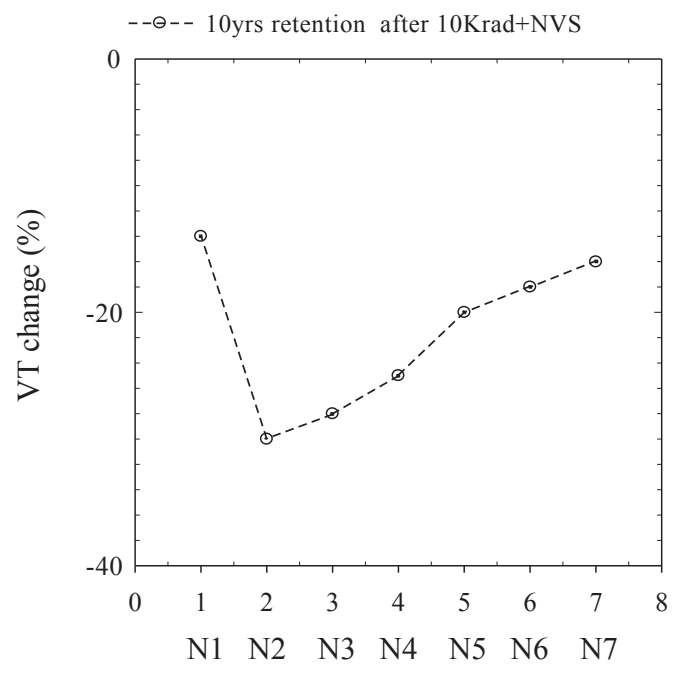

Fig. 8. $V_{\mathrm{T}}$ change with 10 -y retention time under $V_{\mathrm{G}}$ $=-1 \mathrm{~V}$ for various N-TOHOS devices after $10 \mathrm{krad}$ gamma irradiation.



Fig. 10. $V_{\mathrm{T}}$ change with 10 -y retention time under $V_{\mathrm{G}}$ $=-1 \mathrm{~V}$ for various $\mathrm{N}$-TOHOS devices after $5 \mathrm{mrad}$ gamma irradiation.

energy nitrogen-implanted N5-TOHOS devices, the charge retention reliability characteristics after 5 mrad gamma irradiation show the opposite trend with those after $10 \mathrm{krad}$ gamma irradiation. The charge retention reliability characteristic for N7-TOHOS device after 5 mrad gamma irradiation is considerably worse than those for other devices prepared by other implantation methods after 5 mrad gamma irradiation, which is a result of the high-energy-implantation damage of the blocking oxide and the charge leakage path through the blocking oxide after 5 mrad gamma irradiation. 


\section{Conclusions}

The radiation-induced $V_{\mathrm{T}}$ shifts increase with the implantation dose for N-TOHOS devices with low-energy implantation. Furthermore, by applying high-dose and high-energy implantation of nitrogen into $\mathrm{HfO}_{2}$, the charge retention reliability performance is markedly improved after $10 \mathrm{krad}$ gamma irradiation. However, the charge retention reliability characteristic for N7-TOHOS device after $5 \mathrm{mrad}$ gamma irradiation is considerably worse than those for other devices, which is a result of the high-energy-implantation damage of the blocking oxide and the charge leakage path through the blocking oxide after high-dose gamma irradiation. The results show that N-TOHOS with a high implantation dose and a low implantation energy can be a potential candidate nonvolatile TID radiation sensor in the future.

\section{Acknowledgements}

The authors would like to thank the NDL, National Tsing Hua University (NTHU), and National Chiao Tung University (NCTU) for providing the instruments for wafer fabrication and testing. This study was funded in part by the National Science Council (NSC).

\section{References}

1 K. Watanabe, M. Kato, T. Okabe, and M. Nagata: IEEE Trans. Nucl. Sci. 33 (1986) 1216.

2 Y. Takahashi, K. Ohnishi, T. Fujimaki, and M. Yoshikawa: IEEE Trans. Nucl. Sci. 46 (1999) 1578.

3 A. Gasperin, G. Ghidini, A. Cester, and A. Paccagnella: IEEE Trans. Nucl. Sci. 54 (2007) 1898.

4 B. Draper, R. Dockerty, M. Shaneyfelt, S. Habermehl, and J. Murray: IEEE Trans. Nucl. Sci. 55 (2008) 3202.

5 F. Y. Qiao, X. Yu, and L.Y. Pan: Proc. 19th Int. Symp. Phys. Fail. Anal. Integr. Circuits (IPFA) (IEEE, Singapore, 2012) pp. 1-4.

6 W. C. Hsieh, H. T. Lee, and F. C. Jong: Sensors 14 (2014) 14553.

7 Y. Cheng, M. Ding, X. Wu, X. Liu, and K. Wu: 2013 Int. Conf. Solid Dielectrics. (IEEE, Italy, 2013) pp. 764-767.

8 J. G. Park, J. S. Oh, S. D. Yang, K. S. Jeong, Y. M. Kim, H. J. Yun, H. D. Lee, and G. W. Lee: Proc. Nanotechnol. Mater. Devices Conf. (IEEE, California, 2010) pp. 364-367.

9 L. J. Liu, K. S. Chang-Liao, T. Y. Wu, T. K. Wang, W. F. Tsai, and C. F. Ai: Microelectron. Eng. 86 (2009) 1852.

10 B. Y. Tsai,. B. Y. Tsui, P. Li, and C. C. Yen: IEEE EDL. 32 (2011) 1594. 